\title{
Test-Retest Reliability of Isokinetic Knee Strength Measurements in Type 2 Diabetes Mellitus Patients
}

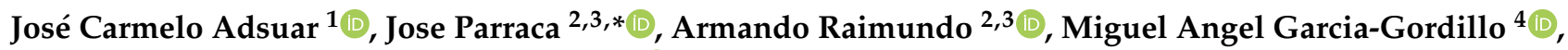 \\ Patricia Polero ${ }^{5}$ and Pablo Tomas-Carus 2,3 (D) \\ 1 Promoting a Healthy Society Research Group, Faculty of Sport Sciences, University of Extremadura, \\ 10003 Cáceres, Spain; jadssal@unex.es \\ 2 Departamento de Desporto e Saúde, Escola de Ciências e Tecnologia, Universidade de Évora, \\ 7000 Évora, Portugal; ammr@uevora.pt (A.R.); ptc@uevora.pt (P.T.-C.) \\ 3 Comprehensive Health Research Centre (CHRC), 7000 Évora, Portugal \\ 4 Departamento de Economía, Universidad Autónoma de Chile, Talca 3460000, Chile; \\ miguel.garcia@uautonoma.cl \\ 5 Laboratorio de Biomecánica y Análisis del Movimiento del Litoral, CENUR Litoral Norte, Universidad de la \\ República, Paysandú 60000, Uruguay; ppoleroc@alumnos.unex.es \\ * Correspondence: jparraca@uevora.pt
}

Citation: Adsuar, J.C.; Parraca, J.; Raimundo, A.; Garcia-Gordillo, M.A.; Polero, P.; Tomas-Carus, P. Test-Retest Reliability of Isokinetic Knee Strength Measurements in Type 2 Diabetes Mellitus Patients. Sustainability 2021, 13, 1343. https://doi.org/10.3390/ su13031343

Academic Editor:

Emilio Villa-González

Received: 16 January 2021

Accepted: 25 January 2021

Published: 28 January 2021

Publisher's Note: MDPI stays neutral with regard to jurisdictional claims in published maps and institutional affiliations.

Copyright: (c) 2021 by the authors. Licensee MDPI, Basel, Switzerland. This article is an open access article distributed under the terms and conditions of the Creative Commons Attribution (CC BY) license (https:/ / creativecommons.org/licenses/by/ $4.0 /)$.

\begin{abstract}
Background: Reliability studies are used to verify the evaluation accuracy of a given device. Strength is an important factor for the development of daily activities and its correct management is fundamental. The objective of this study was to examine the reliability of a concentric strength test in people with type 2 diabetes mellitus (T2DM). Methods: Twenty-seven individuals with T2DM performed three repetitions of extension-flexion in concentric-concentric action at $60^{\circ} / \mathrm{s}$, for both legs, using an isokinetic dynamometer. For the reliability analysis, we performed an intra-session test retest. Results: The total sample and men sub-group intra-class correlation coefficient (ICC) values were excellent for peak torque and work. In the women sub-group, ICC values were excellent for extensors in both peak torque and work; however, concerning flexor, the ICC values were good for peak torque while, for work, they were good for the right leg and moderate for the left leg. Standard error of measurement (SEM) percentage oscillated from 3.85\% to $6.80 \%$, with the smallest real difference (SRD) percentage being from $10.66 \%$ to $18.86 \%$ for peak torque. Furthermore, the SEM (\%) was around 5.5\% and SRD (\%) was around 15\% for work. Conclusions: The isokinetic dynamometry had "good" to "excellent" relative reliability for peak torque (0.862-0.983) and work (0.744-0.982) of extension-flexion in concentric-concentric action at $60^{\circ} / \mathrm{s}$. In addition, our study showed that, in general, an SRD $<20 \%$ could indicate a true change in strength regarding this protocol in T2DM.
\end{abstract}

Keywords: isokinetic strength; type 2 diabetes mellitus; reliability

\section{Introduction}

Diabetes mellitus (DM) is recognized as a dynamic disease that can affect people at different life stages [1]. More specifically, type 2 diabetes mellitus (T2DM) is considered to be a complex disorder that involves different organs of the body (pancreas and insulin system, liver, kidneys, gut, and even the brain) and other body components such as fat cells and muscles [2]. The clinical complications of T2DM are nephropathy, neuropathy, retinopathy, strokes, and coronary heart diseases [3], and diabetes mellitus can result in kidney damage, because toxins in the body cannot be eliminated [4,5]. Furthermore, patients with T2DM may show decreased and lower extremity muscle strength, and often suffer from fatigue, which compromises their ability and willingness to move and can result in motor skills deficits [6-8].

Some biological mechanisms related to T2DM, such as insulin resistance, hyperglycemia, muscle fat infiltration, and oxidative stress, can lead to deterioration of muscle 
strength $[9,10]$. However, one of the mechanisms that influences the loss of muscle strength in T2DM the most is neuropathy [11], possibly due to processes involving motor neurons that show greater and selective atrophy of type Ilb fibers, as observed in diabetic animal muscles $[12,13]$. In this sense, patients with T2DM do not only have lower muscle strength, but also reduced muscle quality (defined as the ratio of strength to the corresponding muscle mass in kilograms) when compared with healthy people [14]. There is ample evidence of a considerable decrease in maximum muscle strength of the lower limbs in patients with diabetes, compared with healthy controls $[15,16]$. Authors have reported reductions in strength of the knee flexor and extensor muscles of $17 \%$ and $14 \%$, respectively [11]. Furthermore, both neuropathy and decreased muscle strength have been related to a reduction of walking activity [17], which can also lead to worse balance and less-efficient gait control [18-20]. Thus, patients with T2DM have an increased risk of falling, due to declines in sensory function and the role of peripheral sensory inputs in the control of gait stability $[19,21]$.

Due to the above, it is essential to assess strength in patients with T2DM, not only in regard to their control of the disease, but also their control of physical conditions and motor abilities in carrying out daily activities, since they depend on muscular strength.

Several studies have performed reliability assessments and quantifications of the isometric, concentric, and eccentric strengths of the knee extensor and flexor muscles using isokinetic dynamometry in healthy individuals [22,23], although some studies have evaluated the strength of the lower limbs of patients with diabetes using an isokinetic dynamometer $[8,14,15]$. Different studies have assessed type 2 diabetics in regards to reliability of accelerometry [24], lower limbs strength [25,26], upper body strength [27], and physical fitness [28]. However, to the best of our knowledge, no previous study to date has assessed reliability, smallest real difference (SRD), or standard measurement error (SEM) regarding knee extensor and flexor muscles in response to T2DM. Reliability allows us to make a correct interpretation of the results of isokinetic dynamometry, since they relate- to the clinician-whether or not there was an authentic change. We are dependent on the consistency of results to provide a relatively low rate or absence of measurement error. The reliability of two moments of assessment (test and retest reliability) are particularly important in the clinical context; a consistent test-retest reliability allows comparisons over time, as well as long-term follow-up. The SRD is a parameter of great importance, since it allows the determination of whether a change observed during a re-evaluation was true and within the limits of the measurement error $[29,30]$. The Sustainable Development Objective 2015-2030 (SDO 3) of the United Nations include 13 goals that have taken into account health-related issues, with SDO 3.4 in particular being related to the control and treatment of non-communicable diseases (NCDs) such as diabetes [31]. Based on this, we deem our study essential for the management of diabetes, as it is essential to evaluate the effectiveness of treatments that aim to improve the strength of the lower body via isokinetic dynamometry in order to achieve the minimum real change. There is a decrease in muscle strength in diabetics [32,33], which can influence the daily activities that involve lower body strength [34].

The present study aims to determine the relative and absolute test-retest reliability of peak torque and work measurements regarding concentric knee extension and flexion in both legs of patients with T2DM by calculating the intraclass correlation coefficient (ICC) for relative reliability and the SRD and SEM for absolute reliability.

\section{Materials and Methods}

\subsection{Participants}

A sample size of 11 participants, with two observations per participant, achieved $92 \%$ power for an intra-class correlation of 0.91 (excellent reliability) under the alternative hypothesis, while under the null hypothesis the intra-class correlation was 0.49 (poor reliability) using an F-test with a significance level of 0.05 . The null and alternative hypotheses were established following the study by Koo et al. [35]. 
A total of 27 participants (12 females and 15 males) were included in the study after verifying that they met the inclusion criteria. Inclusion criteria were: (i) having been diagnosed with T2DM by a physician at least 4 years before beginning the study, (ii) absence of medical problems that would contraindicate or prevent physical loading (e.g., stroke, peripheral vasculopathy, or musculoskeletal injury), (iii) not having been diagnosed with any medical complication as a result of type 2 diabetes such as peripheral neuropathy, nephropathy, glaucoma, or retinopathy, (iv) not being a smoker, (v) not consuming alcohol regularly, (vi) not following any treatment in addition to the usual treatment recommended by the hospital health service, (vii) having an up-to-date medical check-up.

All participants' completed an informed consent form. The local Bioethics and Biosecurity Committee of the University of Évora approved the study protocol (Reference number 08050), which included the tasks reported in the present article. The study was performed following the updates to the Helsinki Declaration, modified by the 64th General Assembly of the World Medical Association (Fortaleza, Brazil, 2013).

\subsection{Measurements}

Several measurements were conducted to characterize the sample. First, the initial questionnaire administered asked participants' ages and the number of years since diagnosis with type 2 diabetes. Second, participants' body weights $(\mathrm{kg})$ were measured without shoes with a calibrate device (Seca 760, Hamburg, Germany), and heights (m) were measured with a calibrate device (Seca 206, Hamburg, Germany), following the international society for the advancement of kinanthropometry (ISAK) [36] international procedure. Body mass index (BMI) was calculated according to this formula: $\mathrm{BMI}=$ weight $(\mathrm{Kg}) /$ height $^{2}(\mathrm{~m})$. Finally, to determine hemoglobin A1C (HbA1C), a blood test was performed following a standardized routine measurement glycated. This hematological parameter could be used to evaluate metabolic control. Optimal control was determined with $\mathrm{HbA1C}$ minor $7 \%$ [37].

Peak torque and work reliability strength regarding knee flexion and extension movements were evaluated using an isokinetic dynamometer (Biodex System-3, Shirley, New York, NY, USA) for both legs. Peak torque was defined as "the single highest torque output recorded throughout the range of motion of each repetition" [38]. The unit of measurement for peak torque is the newton meter $(\mathrm{N} \cdot \mathrm{m})$. Work was defined as "the output of mechanical energy" [39] and represented by the area under the torque versus the angular displacement curve. The measurement unit of work was the Joule (J).

\subsection{Reliability Procedures}

Dynamometer parameters were established following manufacturer instructions: (i) dynamometer orientation was $90^{\circ}$, (ii) dynamometer tilt was $0^{\circ}$, (iii) seat orientation was $90^{\circ}$, (iv) seatback tilt was $85^{\circ}$, (v) axis of rotation was established through the lateral femoral condyle on a sagittal plane, (vi) full flexion was established as the ready position, and (vii) range of movements was $85^{\circ}$. The original software of the isokinetic dynamometer was used to weigh the limb and determine gravity adjustments. Once seated, participants performed the next specific warm-up: three repetitions without load and 1one repetition at $60^{\circ} / \mathrm{s}$. Then, they started the test, which consisted of three repetitions of extension-flexion in concentric-concentric action at $60^{\circ} / \mathrm{s}$. All participants were verbally encouraged to perform as hard as possible throughout the evaluation protocol. All strength assessments were performed by a single evaluator graduated from Sport Sciences with previous experience. In the reliability analyses, the repetition number 2 was used as a "test" and the repetition number 3 was used as a "retest".

\subsection{Measures}

Statistical Analysis

A Shapiro-Wilks test was carried out to check the distribution of data. None of the variables included in the study followed a normal distribution. Data concerning the 
characterization of the sample were given as mean and standard deviation, as well as median and interquartile range. Wilcoxon's test was used to see if there were statistically significant differences between test and retest. The significance level was determined at $p<0.05$.

Reliability was studied through relative reliability and absolute reliability statistics. Relative reliability was determined by the intra-class correlation coefficient $\left(\mathrm{ICC}_{3,1}\right)$ [40]. ICC data were calculated using the following parameters: (i) model: two-way random effects; (ii) type: single rater and; (iii) definition: consistency [35]. Absolute reliability was determined by the standard error of measurement (SEM) and smallest real difference (SRD) [41]. The SEM was calculated with the formula SEM $=\mathrm{SD} \cdot \sqrt{1-I C C}$, where SD is the mean SD of the two repetitions. The SRD formula was SRD $=1.96 \cdot \mathrm{SEM} \cdot \sqrt{2}$. This score was subsequently turned into a percentage.

The following classification was used for interpreting the ICC [42]: an ICC less than 0.5 corresponded to poor reliability, an ICC from 0.5 to 0.75 corresponded to moderate reliability, an ICC from 0.75 to 0.9 corresponded to good reliability, and an ICC greater than 0.9 corresponded to excellent reliability.

Bland-Altman analyses were performed to show the level of agreement between tests and retests regarding peak torque and work. In these graphics, the $x$-axis represented the mean of the test and the $y$-axis showed the difference between the two measurements (A-B; $\mathrm{A}=$ test; $\mathrm{B}=$ retest). Plots showed "the bias" and limits of agreement (LOA) calculated to a $95 \%$ confidence interval. Bias values close to zero represented a strong agreement, and a smaller range between these two LOAs was interpreted as better agreement [43].

\section{Results}

Table 1 includes the ages, anthropometric measurements, body compositions, years of T2DM diagnosis, and levels of glycated hemoglobin for the total sample divided into male and female sub-groups.

Table 1. Characteristics of the study participants.

\begin{tabular}{cccc}
\hline & $\begin{array}{c}\text { Total } \\
(\boldsymbol{n}=\mathbf{2 7}) \\
\text { Mean (SD) }\end{array}$ & $\begin{array}{c}\text { Men } \\
(\boldsymbol{n}=\mathbf{1 5}) \\
\text { Mean (SD) }\end{array}$ & $\begin{array}{c}\text { Women } \\
(\boldsymbol{n}=\mathbf{1 2}) \\
\text { Mean (SD) }\end{array}$ \\
\hline Age (years) & $60.30(6.85)$ & $59.73(6.87)$ & $61(7.07)$ \\
Height $(\mathrm{m})$ & $1.62(0.08)$ & $1.67(0.061)$ & $1.56(0.06)$ \\
Weight $(\mathrm{kg})$ & $82.37(15.12)$ & $87.63(14.92)$ & $75.79(13.12)$ \\
Fat Mass $(\%)$ & $34.89(7.64)$ & $29.80(4.54)$ & $41.23(5.71)$ \\
BMI $\left(\mathrm{kg} / \mathrm{m}^{2}\right)$ & $31.14(4.37)$ & $31.42(4.37)$ & $30.79(4.54)$ \\
Haemoglobin $(\%)$ & $6.89(0.77)$ & $6.63(0.69)$ & $7.21(0.77)$ \\
Years of diagnosis & $10.19(6.71)$ & $8.47(5.59)$ & $12.33(7.58)$ \\
\hline
\end{tabular}

Table 2 shows the descriptive data of the test and retest measurements. Significant differences were observed for all participants regarding peak torque for right knee extensors and flexors, and concerning work for the knee flexors of both legs. In male and female sub-groups, there were only significant differences in peak torque for the right knee flexors.

Table 3 shows the relative reliability (ICC) and absolute reliability (SEM, SEM\%, SRD, and SRD\%). Total sample ICC values were excellent $(>0.95)$ for all variables, both peak torque and work. 
Table 2. Summary of isokinetic peak torque and work.

\begin{tabular}{|c|c|c|c|c|c|c|c|c|c|c|}
\hline \multicolumn{6}{|c|}{ Peak Torque $(\mathbf{N} \cdot \mathrm{m})$} & \multicolumn{5}{|c|}{ Work (J) } \\
\hline & \multicolumn{2}{|c|}{ Day 1} & \multicolumn{3}{|c|}{ Day 2} & \multicolumn{2}{|c|}{ Day 1} & \multicolumn{3}{|c|}{ Day 2} \\
\hline $\begin{array}{c}\text { Test } \\
\text { measurement }\end{array}$ & $\begin{array}{l}\text { Mean } \\
\text { (SD) }\end{array}$ & $\begin{array}{l}\text { Median } \\
\text { (IR) }\end{array}$ & $\begin{array}{l}\text { Mean } \\
\text { (SD) }\end{array}$ & $\begin{array}{l}\text { Median } \\
\text { (IR) }\end{array}$ & $p$ & $\begin{array}{l}\text { Mean } \\
\text { (SD) }\end{array}$ & $\begin{array}{l}\text { Median } \\
\text { (IR) }\end{array}$ & $\begin{array}{l}\text { Mean } \\
\text { (SD) }\end{array}$ & $\begin{array}{l}\text { Median } \\
\text { (IR) }\end{array}$ & $p$ \\
\hline \multicolumn{11}{|l|}{ All participants } \\
\hline $\begin{array}{l}\text { Right leg } \\
\text { extensors }\end{array}$ & $\begin{array}{c}93.80 \\
(49)\end{array}$ & $\begin{array}{c}82.60 \\
(48)\end{array}$ & $\begin{array}{c}97.39 \\
(47)\end{array}$ & $\begin{array}{c}89.20 \\
(38)\end{array}$ & 0.046 & $\begin{array}{c}83.10 \\
(38)\end{array}$ & $\begin{array}{l}71.80 \\
(33)\end{array}$ & $\begin{array}{c}86.11 \\
(37)\end{array}$ & $\begin{array}{c}80.20 \\
(32)\end{array}$ & 0.058 \\
\hline $\begin{array}{l}\text { Left leg } \\
\text { extensors }\end{array}$ & $\begin{array}{c}94.83 \\
(41)\end{array}$ & $\begin{array}{c}83.80 \\
(40)\end{array}$ & $\begin{array}{l}92.84 \\
(42)\end{array}$ & $\begin{array}{c}82.00 \\
(36)\end{array}$ & 0.269 & $\begin{array}{l}85.98 \\
(36)\end{array}$ & $\begin{array}{c}77.00 \\
(32)\end{array}$ & $\begin{array}{c}83.30 \\
(35)\end{array}$ & $\begin{array}{l}70.20 \\
(28)\end{array}$ & 0.080 \\
\hline Right leg flexors & $\begin{array}{c}50.66 \\
(27)\end{array}$ & $\begin{array}{l}40.50 \\
(37)\end{array}$ & $\begin{array}{l}47.51 \\
(26)\end{array}$ & $\begin{array}{l}40.30 \\
(30)\end{array}$ & 0.001 & $\begin{array}{l}48.28 \\
(29)\end{array}$ & $\begin{array}{c}36.50 \\
(40)\end{array}$ & $\begin{array}{c}45.16 \\
(28)\end{array}$ & $\begin{array}{c}38.50 \\
(36)\end{array}$ & 0.025 \\
\hline Left leg flexors & $\begin{array}{c}49.11 \\
(23)\end{array}$ & $\begin{array}{c}43.70 \\
(19)\end{array}$ & $\begin{array}{c}47.74 \\
(23)\end{array}$ & $\begin{array}{c}43.40 \\
(19)\end{array}$ & 0.075 & $\begin{array}{c}49.21 \\
(24)\end{array}$ & $\begin{array}{c}42.50 \\
(18)\end{array}$ & $\begin{array}{c}46.61 \\
(25)\end{array}$ & $\begin{array}{c}41.60 \\
(21)\end{array}$ & 0.035 \\
\hline \multicolumn{11}{|l|}{ Male } \\
\hline $\begin{array}{l}\text { Right leg } \\
\text { extensors }\end{array}$ & $\begin{array}{c}114.20 \\
(55)\end{array}$ & $\begin{array}{c}105.60 \\
(73)\end{array}$ & $\begin{array}{l}116.69 \\
(53)\end{array}$ & $\begin{array}{l}102.50 \\
(59)\end{array}$ & 0.394 & $\begin{array}{c}99.32 \\
(42)\end{array}$ & $\begin{array}{c}87.70 \\
(54)\end{array}$ & $\begin{array}{c}101.59 \\
(41)\end{array}$ & $\begin{array}{c}89.60 \\
(53)\end{array}$ & 0.280 \\
\hline $\begin{array}{l}\text { Left leg } \\
\text { extensors }\end{array}$ & $\begin{array}{c}113.73 \\
(45)\end{array}$ & $\begin{array}{c}104.30 \\
(51)\end{array}$ & $\begin{array}{l}110.60 \\
(47)\end{array}$ & $\begin{array}{c}95.50 \\
(62)\end{array}$ & 0.191 & $\begin{array}{c}101.37 \\
(40)\end{array}$ & $\begin{array}{l}93.10 \\
(39)\end{array}$ & $\begin{array}{c}97.31 \\
(40)\end{array}$ & $\begin{array}{c}90.20 \\
(49)\end{array}$ & 0.149 \\
\hline Right leg flexors & $\begin{array}{c}64.21 \\
(29)\end{array}$ & $\begin{array}{c}57.90 \\
(44)\end{array}$ & $\begin{array}{c}60.84 \\
(27)\end{array}$ & $\begin{array}{c}56.80 \\
(46)\end{array}$ & 0.023 & $\begin{array}{c}60.73 \\
(34)\end{array}$ & $\begin{array}{c}53.10 \\
(60)\end{array}$ & $\begin{array}{c}57.14 \\
(31)\end{array}$ & $\begin{array}{c}54.20 \\
(44)\end{array}$ & 0.100 \\
\hline Left leg flexors & $\begin{array}{c}60.36 \\
(24)\end{array}$ & $\begin{array}{c}52.60 \\
(29)\end{array}$ & $\begin{array}{c}59.30 \\
(25)\end{array}$ & $\begin{array}{c}46.80 \\
(30)\end{array}$ & 0.307 & $\begin{array}{c}59.81 \\
(27)\end{array}$ & $\begin{array}{c}53.10 \\
(30)\end{array}$ & $\begin{array}{c}57.20 \\
(29)\end{array}$ & $\begin{array}{c}46.20 \\
(27)\end{array}$ & 0.124 \\
\hline \multicolumn{11}{|l|}{ Female } \\
\hline $\begin{array}{l}\text { Right leg } \\
\text { extensors }\end{array}$ & $\begin{array}{c}68.29 \\
(24)\end{array}$ & $\begin{array}{c}64.90 \\
(27)\end{array}$ & $\begin{array}{c}73.25 \\
(25)\end{array}$ & $\begin{array}{c}73.30 \\
(42)\end{array}$ & 0.060 & $\begin{array}{c}62.83 \\
(19)\end{array}$ & $\begin{array}{c}64.85 \\
(24)\end{array}$ & $\begin{array}{c}66.76 \\
(19)\end{array}$ & $\begin{array}{c}65.00 \\
(33)\end{array}$ & 0.136 \\
\hline $\begin{array}{l}\text { Left leg } \\
\text { extensors }\end{array}$ & $\begin{array}{c}71.22 \\
(19)\end{array}$ & $\begin{array}{c}70.50 \\
(28)\end{array}$ & $\begin{array}{c}70.64 \\
(18)\end{array}$ & $\begin{array}{c}73.55 \\
(24)\end{array}$ & 0.814 & $\begin{array}{c}66.74 \\
(15)\end{array}$ & $\begin{array}{c}66.65 \\
(22)\end{array}$ & $\begin{array}{c}65.77 \\
(15)\end{array}$ & $\begin{array}{c}66.25 \\
(23)\end{array}$ & 0.456 \\
\hline Right leg flexors & $\begin{array}{c}33.72 \\
(9)\end{array}$ & $\begin{array}{c}34.45 \\
(14)\end{array}$ & $\begin{array}{c}30.84 \\
(10)\end{array}$ & $\begin{array}{c}33.00 \\
(19)\end{array}$ & 0.031 & $\begin{array}{c}32.73 \\
(9)\end{array}$ & $\begin{array}{c}33.35 \\
(14)\end{array}$ & $\begin{array}{c}30.18 \\
(11)\end{array}$ & $\begin{array}{c}31.80 \\
(19)\end{array}$ & 0.155 \\
\hline Left leg flexors & $\begin{array}{c}35.04 \\
(8)\end{array}$ & $\begin{array}{c}35.70 \\
(14)\end{array}$ & $\begin{array}{c}33.30 \\
(10)\end{array}$ & $\begin{array}{c}34.75 \\
(15)\end{array}$ & 0.158 & $\begin{array}{c}35.97 \\
(8)\end{array}$ & $\begin{array}{c}38.95 \\
(11)\end{array}$ & $\begin{array}{c}33.37 \\
(10)\end{array}$ & $\begin{array}{c}32.20 \\
(15)\end{array}$ & 0.170 \\
\hline
\end{tabular}

SD: Standard Deviation; IR: Interquartile Range; N.m: Newton·meter; J: Joules.

Table 3. Test-retest reliability of peak torque and work in two measurements with an interval of days between measurements.

\begin{tabular}{|c|c|c|c|c|c|c|c|c|c|c|}
\hline \multirow{2}{*}{$\begin{array}{c}\text { All }(n=27) \\
\text { Assessed } \\
\text { Action }\end{array}$} & \multicolumn{5}{|c|}{ Peak Torque $(\mathbf{N} \cdot \mathbf{m})$} & \multicolumn{5}{|c|}{ Work (J) } \\
\hline & ICC $(95 \% \mathrm{CI})$ & $\begin{array}{l}\text { SEM } \\
(\mathrm{N} \cdot \mathrm{m})\end{array}$ & $\begin{array}{c}\text { SEM } \\
(\%)\end{array}$ & $\begin{array}{l}\text { SRD } \\
(\mathbf{N} \cdot \mathbf{m})\end{array}$ & $\begin{array}{l}\text { SRD } \\
(\%)\end{array}$ & ICC (95\% CI) & $\begin{array}{c}\text { SEM } \\
\text { (J) }\end{array}$ & $\begin{array}{l}\text { SEM } \\
(\%)\end{array}$ & $\begin{array}{c}\text { SRD } \\
\text { (J) }\end{array}$ & $\begin{array}{l}\text { SRD } \\
(\%)\end{array}$ \\
\hline $\begin{array}{l}\text { Right leg } \\
\text { extensors }\end{array}$ & $\begin{array}{c}0.980 \\
(0.957-0.991)\end{array}$ & 48.12 & 6.80 & 7.1 & 18.86 & $\begin{array}{c}0.977 \\
(0.951-0.989)\end{array}$ & 37.42 & 5.67 & 6.7 & 15.73 \\
\hline $\begin{array}{l}\text { Left leg } \\
\text { extensors }\end{array}$ & $\begin{array}{c}0.978 \\
(0.952-0.990)\end{array}$ & 41.54 & 6.16 & 6.6 & 17.08 & $\begin{array}{c}0.975 \\
(0.947-0.989)\end{array}$ & 35.21 & 5.57 & 6.6 & 15.43 \\
\hline $\begin{array}{l}\text { Right leg } \\
\text { flexors }\end{array}$ & $\begin{array}{c}0.978 \\
(0.953-0.990)\end{array}$ & 26.50 & 3.93 & 8.0 & 10.89 & $\begin{array}{c}0.964 \\
(0.923-0.983)\end{array}$ & 28.52 & 5.41 & 11.6 & 15.00 \\
\hline Left leg flexors & $\begin{array}{c}0.972 \\
(0.940-0.987)\end{array}$ & 22.99 & 3.85 & 7.9 & 10.66 & $\begin{array}{c}0.956 \\
(0.908-0.980)\end{array}$ & 24.57 & 5.15 & 10.8 & 14.28 \\
\hline \multicolumn{11}{|l|}{$\operatorname{Men}(n=15)$} \\
\hline $\begin{array}{l}\text { Right leg } \\
\text { extensors }\end{array}$ & $\begin{array}{c}0.983 \\
(0.950-0.994)\end{array}$ & 53.75 & 7.01 & 6.1 & 19.42 & $\begin{array}{c}0.982 \\
(0.948-0.994)\end{array}$ & 41.45 & 5.56 & 5.5 & 15.41 \\
\hline $\begin{array}{l}\text { Left leg } \\
\text { extensors }\end{array}$ & $\begin{array}{c}0.976 \\
(0.932-0.992)\end{array}$ & 46.09 & 7.14 & 6.4 & 19.79 & $\begin{array}{c}0.974 \\
(0.926-0.991)\end{array}$ & 40.07 & 6.46 & 6.5 & 17.91 \\
\hline $\begin{array}{l}\text { Right leg } \\
\text { flexors }\end{array}$ & $\begin{array}{c}0.977 \\
(0.935-0.992)\end{array}$ & 28.22 & 4.28 & 6.8 & 11.86 & $\begin{array}{c}0.962 \\
(0.895-0.987)\end{array}$ & 32.77 & 6.39 & 10.8 & 17.70 \\
\hline Left leg flexors & $\begin{array}{c}0.970 \\
(0.915-0.990)\end{array}$ & 24.64 & 4.27 & 7.1 & 11.83 & $\begin{array}{c}0.961 \\
(0.891-0.987)\end{array}$ & 28.05 & 5.54 & 9.5 & 15.35 \\
\hline
\end{tabular}


Table 3. Cont.

\begin{tabular}{|c|c|c|c|c|c|c|c|c|c|c|}
\hline \multirow{2}{*}{$\begin{array}{c}\text { All }(n=27) \\
\text { Assessed } \\
\text { Action }\end{array}$} & \multicolumn{5}{|c|}{ Peak Torque $(\mathrm{N} \cdot \mathrm{m})$} & \multicolumn{5}{|c|}{ Work (J) } \\
\hline & ICC $(95 \%$ CI) & $\begin{array}{l}\text { SEM } \\
(\mathbf{N} \cdot \mathbf{m})\end{array}$ & $\begin{array}{l}\text { SEM } \\
(\%)\end{array}$ & $\begin{array}{l}\text { SRD } \\
(\mathbf{N} \cdot \mathbf{m})\end{array}$ & $\begin{array}{l}\text { SRD } \\
(\%)\end{array}$ & ICC $(95 \%$ CI $)$ & $\begin{array}{c}\text { SEM } \\
(\mathrm{J})\end{array}$ & $\begin{array}{c}\text { SEM } \\
(\%)\end{array}$ & $\begin{array}{c}\text { SRD } \\
(J)\end{array}$ & $\begin{array}{l}\text { SRD } \\
(\%)\end{array}$ \\
\hline \multicolumn{11}{|l|}{$\begin{array}{l}\text { Women } \\
(n=12)\end{array}$} \\
\hline $\begin{array}{l}\text { Right leg } \\
\text { extensors }\end{array}$ & $\begin{array}{c}0.931 \\
(0.789-0.979)\end{array}$ & 24.20 & 6.36 & 9.0 & 17.62 & $\begin{array}{c}0.907 \\
(0.722-0.972)\end{array}$ & 18.74 & 5.71 & 8.8 & 15.84 \\
\hline $\begin{array}{l}\text { Left leg } \\
\text { extensors }\end{array}$ & $\begin{array}{c}0.934 \\
(0.796-0.980)\end{array}$ & 18.51 & 4.75 & 6.7 & 13.18 & $\begin{array}{c}0.928 \\
(0.779-0.978)\end{array}$ & 14.89 & 4.00 & 6.0 & 11.07 \\
\hline $\begin{array}{l}\text { Right leg } \\
\text { flexors }\end{array}$ & $\begin{array}{c}0.871 \\
(0.627-0.961)\end{array}$ & 9.49 & 3.41 & 10.6 & 9.45 & $\begin{array}{c}0.835 \\
(0.541-0.949)\end{array}$ & 9.76 & 3.78 & 12.0 & 10.47 \\
\hline Left leg flexors & $\begin{array}{c}0.862 \\
(0.606-0.958)\end{array}$ & 8.71 & 3.24 & 9.5 & 8.97 & $\begin{array}{c}0.744 \\
(0.344-0.918)\end{array}$ & 8.94 & 4.52 & 13.0 & 12.53 \\
\hline
\end{tabular}

ICC: Intra-class Correlation Coefficient; CI: Confidence Interval; SEM: Standard Error Measurement SRD: Small Real Difference N.m: Newton·meter; J: Joules; \%: percentage.

The ICC was slightly better for peak torque than for work. SEM\% oscillated from $3.85 \%$ to $6.80 \%$ and SRD $\%$ from $10.66 \%$ to $18.86 \%$ for peak torque. Furthermore, SEM $\%$ were around $5.5 \%$ and SRD\% were around $15 \%$ for work.

In the male sub-group, ICC values were excellent $(>0.96)$ for all variables, both peak torque and work. Peak torque SEM\% were around $7 \%$ for extensors and $4 \%$ for flexors. Peak torque SRD\% were around $19.5 \%$ for extensors and $12 \%$ for flexors. Furthermore, SEM $\%$ were around $6 \%$ and SRD\% oscillated between $15.35 \%$ to $17.91 \%$ for work.

In the female sub-group, ICC values were excellent $(>0.90)$ for extensors in both peak torque and work. However, for flexor, the ICC values were good regarding peak torque (0.75 to 0.9 ) and, concerning work, they were good for the right leg and moderate for the left leg (0.50 to 0.75$)$.

Figure 1 shows the Bland-Altman plots' peak torques. Bland-Altman plots indicated that the points outside the $95 \%$ LOA were less than $8 \%$ (extensors $7.4 \%$ and flexors $3.7 \%$ ).
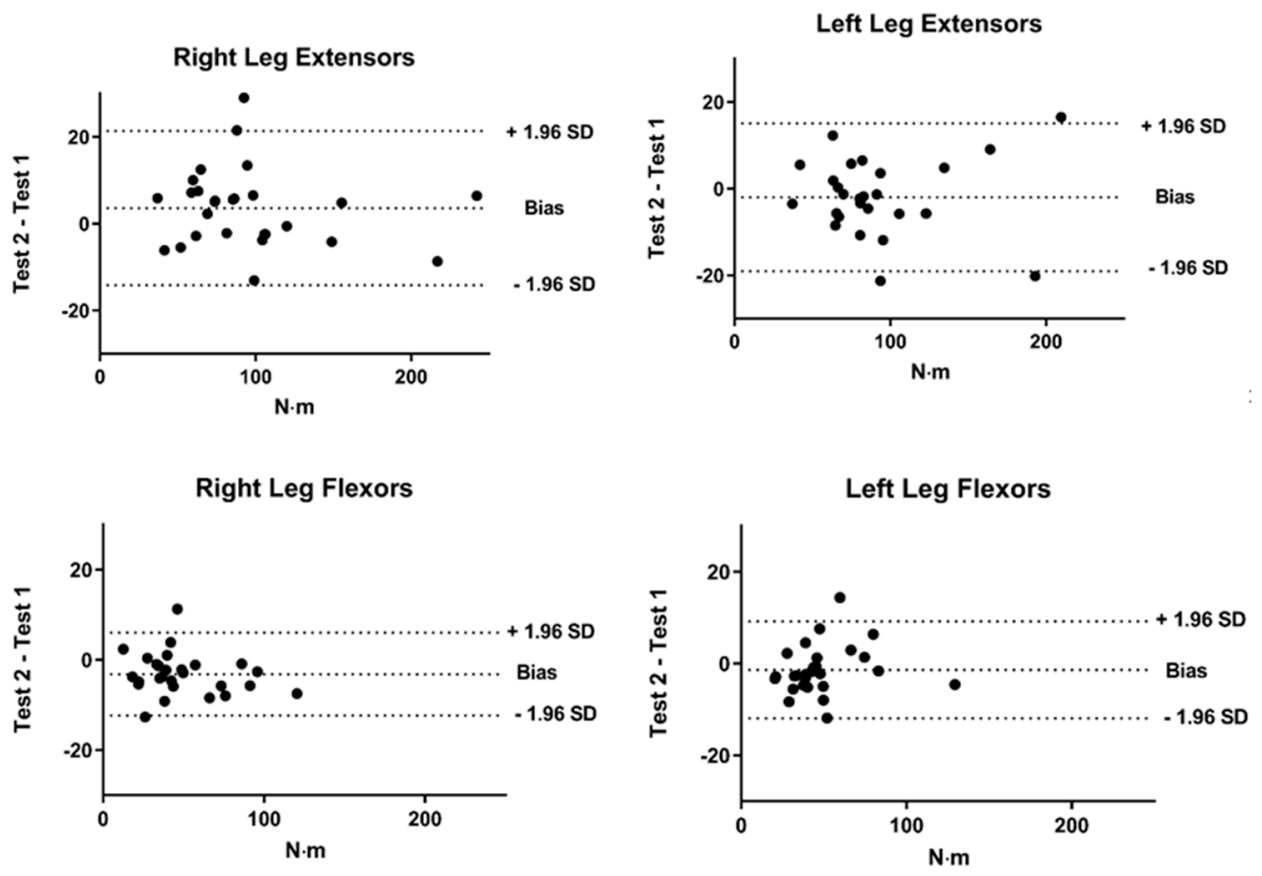

Figure 1. Bland-Altman plots with the bias and the limits of agreement for peak torque for all participants. Upper left corner: right leg extensors; upper right corner: left leg extensors; lower left corner: right leg flexors; lower right corner: left leg flexors. 
Figure 2 shows Bland-Altman plots' work. Bland-Altman plots indicated that the points outside the $95 \%$ LOA were less than $6 \%$ (extensors $5.6 \%$ and flexors $3.7 \%$ ).

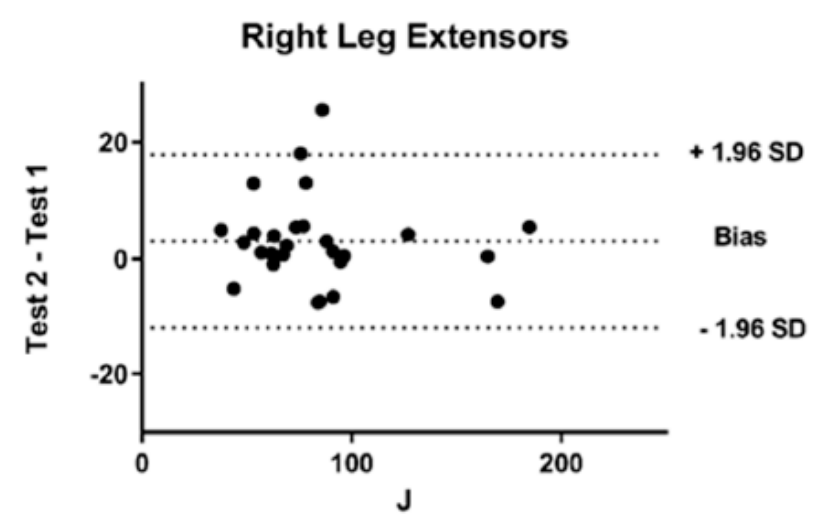

Right Leg Flexors

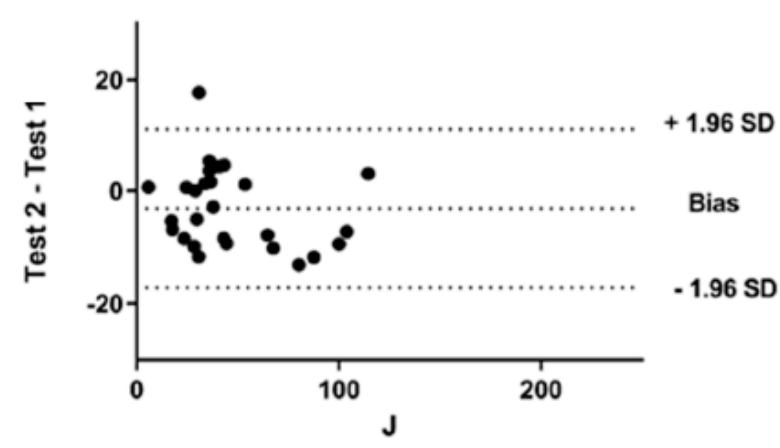

Left Leg Extensors

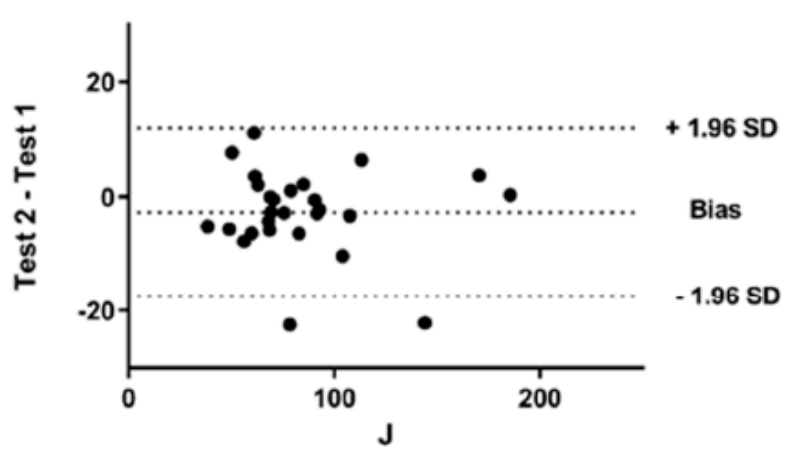

Left Leg Flexors

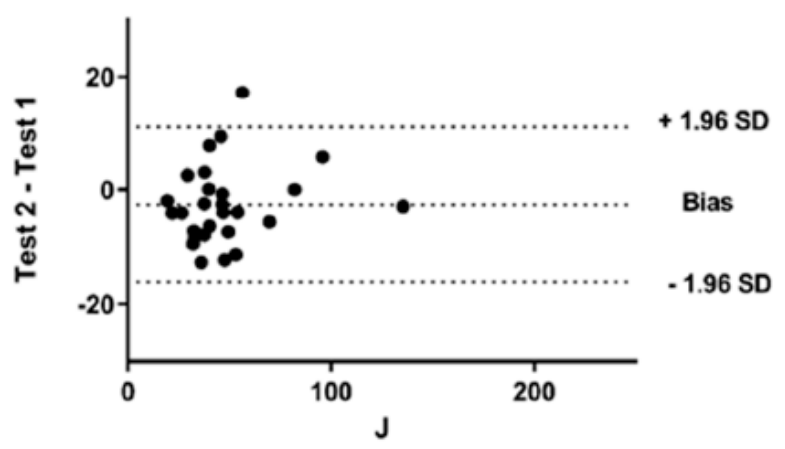

Figure 2. Bland-Altman plots with the bias and the limits of agreement for work for all participants. Upper left corner: right leg extensors; upper right corner: left leg extensors; lower left corner: right leg flexors; lower right corner: left leg flexors.

\section{Discussion}

According to our best knowledge, reliability of three repetitions of extension-flexion in concentric-concentric action at $60^{\circ} / \mathrm{s}$ has not been previously reported in regards to T2DM. Our findings also showed "good" to "excellent" reliability for peak torque and work measurements. In addition, our results showed that, in general, an SRD $<20 \%$ can be considered as a true change in strength concerning this protocol.

The values found regarding relative reliability (ICC) for peak torque ranged from "good" to "excellent". These results agreed with previous studies that evaluated people without pathology [23,44,45]; people with pathologies such as fibromyalgia [29], poststroke hemiparesis [46], and osteoarthritis [47]; and people from all ages, from children [48] to the elderly [49,50]. The values found respecting ICC for work range were all from "good" to "excellent", except for women's left leg flexors. These results matched those of previous studies $[46,47,50]$.

How can the absolute reliability data obtained in this article help us? When carrying out an intervention, not only must we take into account if there are statistically significant differences in the treatment effect to know if it has been effective, but it is fundamental to know if this change can be considered real. No change below SEM can be considered real. Changes between SEM and SRD should be taken with caution, as they could be real with $68 \%$ confidence, while those above SRD could be considered real changes with $95 \%$ confidence. Thanks to the data obtained in this study, it can be concluded that a peak torque change percentage less than the SEM\% (extensors of the right leg: 6.80\%; extensors of the left knee: $6.16 \%$; flexors of the right knee: $3.93 \%$; left knee flexors: $3.85 \%$ ) after an intra-session intervention should not be considered a real change, since it represents a 
change within the variability of the measure. A peak torque difference after an intra-session intervention that is between the SEM of the measurement and the SRD is less likely to be real, standing between $68 \%$ and $95 \%$ confidence (extensors of the right leg: between $6.80 \%$ and $18.86 \%$; left knee extensors: between $6.16 \%$ and 17.08 ; right knee flexors: between $3.93 \%$ and $10.89 \%$; left knee flexors: between $3.85 \%$ and $10.66 \%$ ). When the peak torque values are between the SEM and the SRD, it is necessary to make a clinical decision based on other aspects such as prior familiarity with the test procedures [23]. For example, in a previous article [49] a treatment effect of about a $15 \%$ improvement was found in peak torque leg extensor strength after 12 weeks of combined exercise. This change was between SEM and SRD and should be taken with caution, as it could be considered real at $68 \%$ confidence. However, the treatment effect concerning the peak torque knee flexor intervention was between $24 \%$ and $30 \%$ for the left and right leg, respectively; in this case, we could say that there was an improvement of $14 \%$ in the left leg (treatment effect: SRD; $24 \%-10.66 \%$ $=13.34 \%$ ) and $19 \%$ in the right leg (treatment effect: SRD; $30 \%-10.89 \%=19.11 \%$ ) with $95 \%$ confidence. However, it should be taken into consideration that the present reliability study is an intra-session study and, considering that the study by Tomas-Carus et al. [51] was a longitudinal intervention, it is necessary to carry out an inter-session reliability study in order to make a correct interpretation of the peak toque treatment effect. According to our best knowledge, no study yet been published.

Work percentage changed less than the SEM\% (extensors of the right leg: 5.67\%; extensors of the left knee: $5.57 \%$; flexors of the right knee: $5.41 \%$; left knee flexors: $5.15 \%$ ) after an intra-session intervention; this could not be considered a real change, since it was a change within the variability of the measure. A work difference after an intra-session intervention that is between the SEM of the measurement and the SRD is less likely to be real, situated between $68 \%$ and $95 \%$ confidence (extensors of the right leg: between $5.67 \%$ and $15.73 \%$; left knee extensors: between $5.57 \%$ and $15.43 \%$; right knee flexors: between $5.41 \%$ and $15 \%$; left knee flexors: between $5.15 \%$ and $14.28 \%$ ). When the work values are between the SEM and the SRD, it is necessary to make a clinical decision based on other aspects such as prior familiarity with the test procedures [23]. Values above the SRD can be considered a real change (extensors of the right leg: $>15.73 \%$; left knee extensors: $>15.43 \%$; right knee flexors: $>15 \%$; left knee flexors: $>14.28 \%$ ).

Visual inspection using Bland-Altman plots for peak torque and work did not show signs of any systematic bias in the differences between tests and retests.

Some potential limitations need to be addressed. Despite having a sample size greater than the minimum required, the small sample size could have contributed to decreased statistical power in some variables, and neither the volume nor the intensity of the participants' physical activity were controlled. However, our results showed excellent reliability in most of the variables measured.

On the other hand, the risk of measurement bias must also be considered due to: (i) alignment of the mechanical axis of the isokinetic dynamometer to the knee was performed manually with a visual inspection, which may have contributed to a slight difference in placement between test and retest; (ii) despite encouraging participants to perform the test and re-retest to their maximum ability, it is difficult to determine if the participants performed each repetition to their full potential unless an electromyogram is done simultaneously; and (iii) only one angular velocity $\left(60^{\circ} / \mathrm{s}\right)$ and muscular contraction (concentric) were used. Thus, more studies are needed to investigate the test-retest reliability of other angular velocities and muscular actions that might be considered clinically relevant and provide normative data that can be used to more accurately assess treatments involving muscle strength training in patients with T2DM.

\section{Conclusions}

To the best of our knowledge, this is the first study in reliability, smallest real difference (SRD), or standard measurement error (SEM) regarding knee extensor and flexor muscles at $60^{\circ} / \mathrm{s}$ in T2DM showing excellent and good/moderate reliability for peak torque and work. 
These results could indicate that isokinetic dynamometry provides reliable measurements of peak torque and work regarding concentric extension and concentric flexion in patients with T2DM.

Author Contributions: Formal analysis, A.R.; Supervision, P.P.; Writing—original draft, J.C.A., J.P. and P.T.-C.; Writing—review \& editing, M.A.G.-G. All authors have read and agreed to the published version of the manuscript.

Funding: This research was funded by UÉvora—UniverCIDADE VI—Contrato-Programa de Desenvolvimento Desportivo n.o CP/534/DDT/2020. IPDJ, I.P.

Institutional Review Board Statement: The study was conducted according to the guidelines of the Declaration of Helsinki, and approved by the Ethics Committee of Universidade de Évora (protocol code 08050 approved on $09 / 25 / 2008$ ).

Informed Consent Statement: Informed consent was obtained from all subjects involved in the study.

Acknowledgments: Acknowledgements to Family Health Unit Lusitania-Évora, in special to Vanda Lapão Silva and the nursing team (Arlinda Marques, Luísa Patricio and Leonor Ricardo) for the all the support provided during the preparation of the study.

Conflicts of Interest: The authors declare no conflict of interest.

\section{References}

1. The Lancet. Diabetes: A dynamic disease. Lancet 2017, 389, 2163. [CrossRef]

2. Brunton, S. Pathophysiology of Type 2 Diabetes: The Evolution of Our Understanding. J. Fam. Pract. 2016, 65, 4.

3. Zheng, Y.; Ley, S.H.; Hu, F.B. Global aetiology and epidemiology of type 2 diabetes mellitus and its complications. Nat. Rev. Endocrinol. 2018, 14, 88-98. [CrossRef]

4. Andrade-Guel, M.; Cabello-Alvarado, C.; Cruz-Delgado, V.J.; Bartolo-Perez, P.; De Leon-Martinez, P.A.; Saenz-Galindo, A.; Cadenas-Pliego, G.; Avila-Orta, C.A. Surface Modification of Graphene Nanoplatelets by Organic Acids and Ultrasonic Radiation for Enhance Uremic Toxins Adsorption. Materials 2019, 12, 715. [CrossRef]

5. Cabello-Alvarado, C.; Andrade-Guel, M.; Perez-Alvarez, M.; Cadenas-Pliego, G.; Cortes-Hernandez, D.A.; Bartolo-Perez, P.; Avila-Orta, C.A.; Cruz-Delgado, V.J.; Zepeda-Pedreguera, A. Graphene Nanoplatelets Modified with Amino-Groups by Ultrasonic Radiation of Variable Frequency for Potential Adsorption of Uremic Toxins. Nanomaterials 2019, 9, 1261. [CrossRef]

6. Andersen, H. Motor dysfunction in diabetes. Diabetes Metab. Res. Rev. 2012, 28 (Suppl. S1), 89-92. [CrossRef]

7. Nomura, T.; Kawae, T.; Kataoka, H.; Ikeda, Y. Aging, physical activity, and diabetic complications related to loss of muscle strength in patients with type 2 diabetes. Phys. Ther. Res. 2018, 21, 33-38. [CrossRef]

8. Park, S.W.; Goodpaster, B.H.; Strotmeyer, E.S.; Kuller, L.H.; Broudeau, R.; Kammerer, C.; de Rekeneire, N.; Harris, T.B.; Schwartz, A.V.; Tylavsky, F.A.; et al. Accelerated loss of skeletal muscle strength in older adults with type 2 diabetes: The health, aging, and body composition study. Diabetes Care 2007, 30, 1507-1512. [CrossRef]

9. Bianchi, L.; Volpato, S. Muscle dysfunction in type 2 diabetes: A major threat to patient's mobility and independence. Acta Diabetol. 2016, 53, 879-889. [CrossRef]

10. Tsutsui, H.; Kinugawa, S.; Matsushima, S.; Yokota, T. Oxidative stress in cardiac and skeletal muscle dysfunction associated with diabetes mellitus. J. Clin. Biochem. Nutr. 2011, 48, 68-71. [CrossRef]

11. Andersen, H.; Nielsen, S.; Mogensen, C.E.; Jakobsen, J. Muscle strength in type 2 diabetes. Diabetes 2004, 53, 1543-1548. [CrossRef] [PubMed]

12. Klueber, K.M.; Feczko, J.D.; Schmidt, G.; Watkins, J.B., 3rd. Skeletal muscle in the diabetic mouse: Histochemical and morphometric analysis. Anat. Rec. 1989, 225, 41-45. [CrossRef] [PubMed]

13. Medina-Sanchez, M.; Rodriguez-Sanchez, C.; Vega-Alvarez, J.A.; Menedez-Pelaez, A.; Perez-Casas, A. Proximal skeletal muscle alterations in streptozotocin-diabetic rats: A histochemical and morphometric analysis. Am. J. Anat. 1991, 191, 48-56. [CrossRef]

14. Park, S.W.; Goodpaster, B.H.; Strotmeyer, E.S.; de Rekeneire, N.; Harris, T.B.; Schwartz, A.V.; Tylavsky, F.A.; Newman, A.B. Decreased muscle strength and quality in older adults with type 2 diabetes: The health, aging, and body composition study. Diabetes 2006, 55, 1813-1818. [CrossRef]

15. TH, I.J.; Schaper, N.C.; Melai, T.; Meijer, K.; Willems, P.J.; Savelberg, H.H. Lower extremity muscle strength is reduced in people with type 2 diabetes, with and without polyneuropathy, and is associated with impaired mobility and reduced quality of life. Diabetes Res. Clin. Pract. 2012, 95, 345-351. [CrossRef]

16. Ferreira, J.P.; Sartor, C.D.; Leal, A.M.; Sacco, I.C.; Sato, T.O.; Ribeiro, I.L.; Soares, A.S.; Cunha, J.E.; Salvini, T.F. The effect of peripheral neuropathy on lower limb muscle strength in diabetic individuals. Clin. Biomech. 2017, 43, 67-73. [CrossRef] [PubMed] 
17. van Sloten, T.T.; Savelberg, H.H.; Duimel-Peeters, I.G.; Meijer, K.; Henry, R.M.; Stehouwer, C.D.; Schaper, N.C. Peripheral neuropathy, decreased muscle strength and obesity are strongly associated with walking in persons with type 2 diabetes without manifest mobility limitations. Diabetes Res. Clin. Pract. 2011, 91, 32-39. [CrossRef] [PubMed]

18. Strotmeyer, E.S.; de Rekeneire, N.; Schwartz, A.V.; Faulkner, K.A.; Resnick, H.E.; Goodpaster, B.H.; Shorr, R.I.; Vinik, A.I.; Harris, T.B.; Newman, A.B. The relationship of reduced peripheral nerve function and diabetes with physical performance in older white and black adults: The Health, Aging, and Body Composition (Health ABC) study. Diabetes Care 2008, 31, 1767-1772. [CrossRef]

19. Menz, H.B.; Lord, S.R.; St George, R.; Fitzpatrick, R.C. Walking stability and sensorimotor function in older people with diabetic peripheral neuropathy. Arch. Phys. Med. Rehabil. 2004, 85, 245-252. [CrossRef]

20. Petrofsky, J.; Lee, S.; Macnider, M.; Navarro, E. Autonomic, endothelial function and the analysis of gait in patients with type 1 and type 2 diabetes. Acta Diabetol. 2005, 42, 7-15. [CrossRef]

21. Hewston, P.; Deshpande, N. Falls and Balance Impairments in Older Adults with Type 2 Diabetes: Thinking Beyond Diabetic Peripheral Neuropathy. Can. J. Diabetes 2016, 40, 6-9. [CrossRef]

22. Frontera, W.R.; Hughes, V.A.; Dallal, G.E.; Evans, W.J. Reliability of isokinetic muscle strength testing in 45- to 78-year-old men and women. Arch. Phys. Med. Rehabil. 1993, 74, 1181-1185.

23. Sole, G.; Hamren, J.; Milosavljevic, S.; Nicholson, H.; Sullivan, S.J. Test-retest reliability of isokinetic knee extension and flexion. Arch. Phys. Med. Rehabil. 2007, 88, 626-631. [CrossRef]

24. de Bruin, E.D.; Hubli, M.; Hofer, P.; Wolf, P.; Murer, K.; Zijlstra, W. Validity and Reliability of Accelerometer-Based Gait Assessment in Patients with Diabetes on Challenging Surfaces. J. Aging Res. 2012, 2012, 954378. [CrossRef]

25. Barrios-Fernández, S.; Pérez-Gómez, J.; Galán-Arroyo, M.d.C.; Señorán-Rivera, J.; Martín-Carmona, R.; Mendoza-Muñoz, M.; García-Gordillo, M.Á.; Domínguez-Muñoz, F.J.; Adsuar, J.C. Reliability of 30-s Chair Stand Test with and without Cognitive Task in People with Type-2 Diabetes Mellitus. Int. J. Environ. Res. Public Health 2020, 17, 1450. [CrossRef]

26. Dominguez-Muñoz, F.J.; Hernández-Mocholi, M.A.; Manso, L.J.; Collado-Mateo, D.; Villafaina, S.; Adsuar, J.C.; Gusi, N. TestRetest Reliability of Kinematic Parameters of Timed Up and Go in People with Type 2 Diabetes. Appl. Sci. 2019, 9, 4709. [CrossRef]

27. Lexie Wright BSc, D.; Craig Wassinger, P.; Hansson, M.; Johansson, S.; Todd, N. Reliability of hand-held dynamometric strength testing in people with diabetes/chronic conditions. N. Z. J. Physiother. 2010, 38, 52.

28. Alfonso-Rosa, R.M.; del Pozo-Cruz, B.; del Pozo-Cruz, J.; Sañudo, B.; Rogers, M.E. Test-retest reliability and minimal detectable change scores for fitness assessment in older adults with type 2 diabetes. Rehabil. Nurs. 2014, 39, 260-268. [CrossRef]

29. Adsuar, J.C.; Olivares, P.R.; del Pozo-Cruz, B.; Parraca, J.A.; Gusi, N. Test-retest reliability of isometric and isokinetic knee extension and flexion in patients with fibromyalgia: Evaluation of the smallest real difference. Arch. Phys. Med. Rehabil. 2011, 92, 1646-1651. [CrossRef]

30. Meeteren, J.; Roebroeck, M.E.; Stam, H.J. Test-retest reliability in isokinetic muscle strength measurements of the shoulder. J. Rehabil. Med. 2002, 34, 91-95. [CrossRef]

31. Nishtar, S.; Niinistö, S.; Sirisena, M.; Vázquez, T.; Skvortsova, V.; Rubinstein, A.; Mogae, F.G.; Mattila, P.; Ghazizadeh Hashemi, S.H.; Kariuki, S.; et al. Time to deliver: Report of the WHO Independent High-Level Commission on NCDs. Lancet 2018, 392, 245-252. [CrossRef]

32. Andersen, H.; Poulsen, P.L.; Mogensen, C.E.; Jakobsen, J. Isokinetic muscle strength in long-term IDDM patients in relation to diabetic complications. Diabetes 1996, 45, 440-445. [CrossRef]

33. Hatef, B.; Ghanjal, A.; Meftahi, G.H.; Askary-Ashtiani, A. Isokinetic and Electromyographic Properties of Muscular Endurance in Short and Long-Term Type 2 Diabetes. Glob. J. Health Sci. 2016, 8, 210-219. [CrossRef]

34. Volpato, S.; Maraldi, C.; Fellin, R. Type 2 diabetes and risk for functional decline and disability in older persons. Curr. Diabetes Rev. 2010, 6, 134-143. [CrossRef]

35. Koo, T.K.; Li, M.Y. A Guideline of Selecting and Reporting Intraclass Correlation Coefficients for Reliability Research. J. Chiropr. Med. 2016, 15, 155-163. [CrossRef]

36. Marfell-Jones, M.J.; Stewart, A.; De Ridder, J. International Standards for Anthropometric Assessment; International Society for the Advancement of Kinanthropometry: Wellington, New Zealand, 2012.

37. Grey, M.; Boland, E.A.; Davidson, M.; Li, J.; Tamborlane, W.V. Coping skills training for youth with diabetes mellitus has long-lasting effects on metabolic control and quality of life. J. Pediatr. 2000, 137, 107-113. [CrossRef]

38. Perrin, D.H.; Robertson, R.J.; Ray, R.L. Bilateral isokinetic peak torque, torque acceleration energy, power, and work relationships in athletes and nonathletes. J. Orthop. Sports Phys. Ther. 1987, 9, 184-189. [CrossRef]

39. Le-Ngoc, L.; Janssen, J. Validity and reliability of a hand-held dynamometer for dynamic muscle strength assessment. Rehabil. Med. 2012, 4, 53-66.

40. Shrout, P.E.; Fleiss, J.L. Intraclass correlations: Uses in assessing rater reliability. Psychol. Bull. 1979, 86, 420-428. [CrossRef]

41. Weir, J.P. Quantifying test-retest reliability using the intraclass correlation coefficient and the SEM. J. Strength Cond. Res. 2005, 19, 231-240.

42. Portney, L.; Watkins, M. Construct Validity. Foundations of Clinical Research: Applications to Practice; Prentice Hall Health: Upper Saddle River, NJ, USA, 2000; pp. 87-91.

43. Bland, J.M.; Altman, D.G. Statistical methods for assessing agreement between two methods of clinical measurement. Lancet 1986, 1, 307-310. [CrossRef] 
44. Phillips, B.A.; Lo, S.K.; Mastaglia, F.L. Isokinetic and isometric torque values using a Kin-Com dynamometer in normal subjects aged 20 to 69 years. Isokinet. Exerc. Sci. 2000, 8, 147-159. [CrossRef]

45. Harding, B.; Black, T.; Bruulsema, A.; Maxwell, B.; Stratford, P. Reliability of a reciprocal test protocol performed on the kinetic communicator: An isokinetic test of knee extensor and flexor strength. J. Orthop. Sports Phys. Ther. 1988, 10, 218-223. [CrossRef]

46. Dehkordi, S.N.; Talebian, S.; Olyaei, G.; Montazeri, A. Reliability of isokinetic normalized peak torque assessments for knee muscles in post-stroke hemiparesis. Gait Posture 2008, 27, 715-718. [CrossRef]

47. Carpenter, M.; Carpenter, R.; Peel, J.; Zukley, L. The reliability of isokinetic and isometric leg strength measures among individuals with symptoms of mild osteoarthritis. J. Sports Med. Phys. Fit. 2006, 46, 585. [CrossRef]

48. Muñoz-Bermejo, L.; Pérez-Gómez, J.; Manzano, F.; Collado-Mateo, D.; Villafaina, S.; Adsuar, J.C. Reliability of isokinetic knee strength measurements in children: A systematic review and meta-analysis. PLoS ONE 2019, 14, e0226274. [CrossRef]

49. Symons, T.B.; Vandervoort, A.A.; Rice, C.L.; Overend, T.J.; Marsh, G.D. Reliability of isokinetic and isometric knee-extensor force in older women. J. Aging Phys. Act. 2004, 12, 525-537. [CrossRef]

50. Hartmann, A.; Knols, R.; Murer, K.; De Bruin, E.D. Reproducibility of an isokinetic strength-testing protocol of the knee and ankle in older adults. Gerontology 2009, 55, 259-268. [CrossRef]

51. Tomas-Carus, P.; Ortega-Alonso, A.; Pietilainen, K.H.; Santos, V.; Goncalves, H.; Ramos, J.; Raimundo, A. A randomized controlled trial on the effects of combined aerobic-resistance exercise on muscle strength and fatigue, glycemic control and health-related quality of life of type 2 diabetes patients. J. Sports Med. Phys. Fit. 2016, 56, 572-578. 